\title{
Impact of Multiple-Curve Dynamics in Credit Valuation Adjustments
}

\author{
Giacomo Bormetti, Damiano Brigo, Marco Francischello \\ and Andrea Pallavicini
}

\begin{abstract}
We present a detailed analysis of interest rate derivatives valuation under credit risk and collateral modeling. We show how the credit and collateral extended valuation framework in Pallavicini et al. (2011) can be helpful in defining the key market rates underlying the multiple interest rate curves that characterize current interest rate markets. We introduce the collateralized valuation measures and formulate a consistent realistic dynamics for the rates emerging from our analysis. We point out limitations of multiple curve models with deterministic basis considering valuation of particularly sensitive products such as basis swaps.
\end{abstract}

Keywords Multiple curves - Evaluation adjustments - Basis swaps - Collateral • HJM model

\section{Introduction}

After the onset of the crisis in 2007, all market instruments are quoted by taking into account, more or less implicitly, credit- and collateral-related adjustments. As a consequence, when approaching modeling problems one has to carefully check standard theoretical assumptions which often ignore credit and liquidity issues. One has to go back to market processes and fundamental instruments by limiting oneself to use models based on products and quantities that are available on the market.

\footnotetext{
G. Bormetti $(\bowtie)$

University of Bologna, Piazza di Porta San Donato 5, 40126 Bologna, Italy

e-mail: giacomo.bormetti@unibo.it

D. Brigo $\cdot$ M. Francischello

Imperial College London, London SW7 2AZ, UK

e-mail: damiano.brigo@imperial.ac.uk

M. Francischello

e-mail: m.francischello14@imperial.ac.uk
}

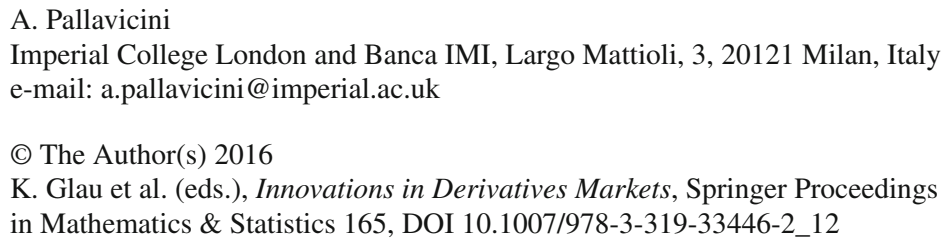


Referring to market observables and processes is the only means we have to validate our theoretical assumptions, so as to drop them if in contrast with observations. This general recipe is what is guiding us in this paper, where we try to adapt interest rate models for valuation to the current landscape.

A detailed analysis of the updated valuation problem one faces when including credit risk and collateral modeling (and further funding costs) has been presented elsewhere in this volume, see for example [6,7]. We refer to those papers and references therein for a detailed discussion. Here we focus our updated valuation framework to consider the following key points: (i) focus on interest rate derivatives; (ii) understand how the updated valuation framework can be helpful in defining the key market rates underlying the multiple interest rate curves that characterize current interest rate markets; (iii) define collateralized valuation measures; (iv) formulate a consistent realistic dynamics for the rates emerging from the above analysis; (v) show how the framework can be applied to valuation of particularly sensitive products such as basis swaps under credit risk and collateral posting;(vi) point out limitations in some current market practices such as explaining the multiple curves through deterministic fudge factors or shifts where the option embedded in the credit valuation adjustment (CVA) calculation would be priced without any volatility. For an extended version of this paper we remand to [3]. This paper is an extended and refined version of ideas originally appeared in [24].

\section{Valuation Equation with Credit and Collateral}

Classical interest-rate models were formulated to satisfy no-arbitrage relationships by construction, which allowed one to price and hedge forward-rate agreements in terms of risk-free zero-coupon bonds. Starting from summer 2007, with the spreading of the credit crunch, market quotes of forward rates and zero-coupon bonds began to violate usual no-arbitrage relationships. The main driver of such behavior was the liquidity crisis reducing the credit lines along with the fear of an imminent systemic break-down. As a result the impact of counterparty risk on market prices could not be considered negligible any more.

This is the first of many examples of relationships that broke down with the crisis. Assumptions and approximations stemming from valuation theory should be replaced by strategies implemented with market instruments. For instance, inclusion of CVA for interest-rate instruments, such as those analyzed in [8], breaks the relationship between risk-free zero-coupon bonds and LIBOR forward rates. Also, funding in domestic currency on different time horizons must include counterparty risk adjustments and liquidity issues, see [15], breaking again this relationship. We thus have, against the earlier standard theory,

$$
L\left(T_{0}, T_{1}\right) \neq \frac{1}{T_{1}-T_{0}}\left(\frac{1}{P_{T_{0}}\left(T_{1}\right)}-1\right), \quad F_{t}\left(T_{0}, T_{1}\right) \neq \frac{1}{T_{1}-T_{0}}\left(\frac{P_{t}\left(T_{0}\right)}{P_{t}\left(T_{1}\right)}-1\right)
$$


where $P_{t}(T)$ is a zero-coupon bond price at time $t$ for maturity $T, L$ is the LIBOR rate and $F$ is the related LIBOR forward rate. A direct consequence is the impossibility to describe all LIBOR rates in terms of a unique zero-coupon yield curve. Indeed, since 2009 and even earlier, we had evidence that the money market for the Euro area was moving to a multi-curve setting. See [1, 19, 20, 27].

\subsection{Valuation Framework}

In order to value a financial product (for example a derivative contract), we have to discount all the cash flows occurring after the trading position is entered. We follow the approach of $[25,26]$ and we specialize it to the case of interest-rate derivatives, where collateralization usually happens on a daily basis, and where gap risk is not large. Hence we prefer to present such results when cash flows are modeled as happening in a continuous time-grid, since this simplifies notation and calculations. We refer to the two names involved in the financial contract and subject to default risk as investor (also called name "I") and counterparty (also called name "C"). We denote by $\tau_{I}$, and $\tau_{C}$, respectively, the default times of the investor and counterparty. We fix the portfolio time horizon $T>0$, and fix the risk-neutral valuation model $(\Omega, \mathscr{G}, \mathbb{Q})$, with a filtration $\left(\mathscr{G}_{t}\right)_{t \in[0, T]}$ such that $\tau_{C}, \tau_{I}$ are $\left(\mathscr{G}_{t}\right)_{t \in[0, T]}$-stopping times. We denote by $\mathbb{E}_{t}[\cdot]$ the conditional expectation under $\mathbb{Q}$ given $\mathscr{G}_{t}$, and by $\mathbb{E}_{\tau_{i}}[\cdot]$ the conditional expectation under $\mathbb{Q}$ given the stopped filtration $\mathscr{G}_{\tau_{i}}$. We exclude the possibility of simultaneous defaults, and define the first default event between the two parties as the stopping time $\tau:=\tau_{C} \wedge \tau_{I}$.

We will also consider the market sub-filtration $\left(\mathscr{F}_{t}\right)_{t \geq 0}$ that one obtains implicitly by assuming a separable structure for the complete market filtration $\left(\mathscr{G}_{t}\right)_{t \geq 0} . \mathscr{G}_{t}$ is then generated by the pure default-free market filtration $\mathscr{F}_{t}$ and by the filtration generated by all the relevant default times monitored up to $t$ (see for example [2]).

We introduce a risk-free rate $r$ associated with the risk-neutral measure. We therefore need to define the related stochastic discount factor $D(t, u, r)$ that in general will denote the risk-neutral default-free discount factor, given by the ratio

$$
D(t, u, r)=B_{t} / B_{u}, \quad d B_{t}=r_{t} B_{t} d t,
$$

where $B$ is the bank account numeraire, driven by the risk-free instantaneous interest rate $r_{t}$ and associated to the risk-neutral measure $\mathbb{Q}$. This rate $r_{t}$ is assumed to be $\left(\mathscr{F}_{t}\right)_{t \in[0, T]}$ adapted and is the key variable in all pre-crisis term structure modeling.

We now want to price a collateralized derivative contract, and in particular we assume that collateral re-hypothecation is allowed, as done in practice (see [4] for a discussion on re-hypothecation). We thus write directly the adjustment payout terms as carry costs cash flows, each accruing at the relevant rate, namely the price $V_{t}$ of a derivative contract, inclusive of collateralized credit and debit risk, margining costs, can be derived by following $[25,26]$, and is given by: 


$$
V_{t}=\mathbb{E}\left[\int_{t}^{T} D(t, u ; r)\left(1_{\{u<\tau\}} d \pi_{u}+1_{\{\tau \in d u\}} \theta_{u}+\left(r_{u}-c_{u}\right) C_{u} d u\right) \mid \mathscr{G}_{t}\right]
$$

where

- $\pi_{u}$ is the coupon process of the product, without credit or debit risk and without collateral cash flows;

- $C_{u}$ is the collateral process, and we use the convention that $C_{u}>0$ while $I$ is the collateral receiver and $C_{u}<0$ when $I$ is the collateral poster. $\left(r_{u}-c_{u}\right) C_{u}$ are the collateral margining costs and the collateral rate is defined as $c_{t}:=c_{t}^{+} 1_{\left\{C_{t}>0\right\}}+$ $c_{t}^{-} 1_{\left\{C_{t}<0\right\}}$ with $c^{ \pm}$defined in the CSA contract. In general we may assume the processes $c^{+}, c^{-}$to be adapted to the default-free filtration $\mathscr{F}_{t}$.

- $\theta_{u}=\theta_{u}(C, \varepsilon)$ is the on-default cash flow process that depends on the collateral process $C_{u}$ and the close-out value $\varepsilon_{u}{ }^{1}$ It is primarily this term that originates the credit and debit valuation adjustments (CVA/DVA) terms, that may also embed collateral and gap risk due to the jump at default of the value of the considered deal (e.g. in a credit derivative), see for example [5].

Notice that the above valuation equation (2) is not suited for explicit numerical evaluations, since the right-hand side is still depending on the derivative price via the indicators within the collateral rates and possibly via the close-out term, leading to recursive/nonlinear features. We could resort to numerical solutions, as in [11], but, since our goal is valuing interest-rate derivatives, we prefer to further specialize the valuation equation for such deals.

\subsection{The Master Equation Under Change of Filtration}

In this first work we develop our analysis without considering a dependence between the default times if not through their spreads, or more precisely by assuming that the default times are $\mathscr{F}$-conditionally independent. Moreover, we assume that the collateral account and the close-out processes are $\mathscr{F}$-adapted. Thus, we can simplify the valuation equation given by (2) by switching to the default-free market filtration. By following the filtration switching formula in [2], we introduce for any $\mathscr{G}_{t}$-adapted process $X_{t}$ a unique $\mathscr{F}_{t}$-adapted process $\widetilde{X}_{t}$, defined such that $1_{\{\tau>t\}} X_{t}=1_{\{\tau>t\}} \widetilde{X}_{t}$. Hence, we can write the pre-default price process as given by $1_{\{\tau>t\}} \widetilde{V}_{t}=V_{t}$ where the right-hand side is given in Eq. (2) and where $\tilde{V}_{t}$ is $\mathscr{F}_{t}$-adapted. Before changing filtration, we have to specify the form of the close-out payoff:

$$
\theta_{\tau}=\varepsilon_{\tau}(\tau, T)-1_{\left\{\tau_{C}<\tau_{I}\right\}} L G D_{C}\left(\varepsilon_{\tau}(\tau, T)-C_{\tau}\right)^{+}-1_{\left\{\tau_{I}<\tau_{C}\right\}} L G D_{I}\left(\varepsilon_{\tau}(\tau, T)-C_{\tau}\right)^{-}
$$

\footnotetext{
${ }^{1}$ The closeout value is the residual value of the contract at default time and the CSA specifies the way it should be computed.
} 
where $L G D \leq 1$ is the loss given default, $(x)^{+}$indicates the positive part of $x$ and $(x)^{-}=-(-x)^{+}$. For an extended discussion of the term $\theta_{\tau}$ we refer to [3]. Moreover, to derive an explicit valuation formula we assume that gap risk is not present, namely $\tilde{V}_{\tau-}=\tilde{V}_{\tau}$, and we consider a particular form for collateral and close-out prices, namely we model the close-out value as

$$
\varepsilon_{s}(t, T)=\mathbb{E}\left[\int_{t}^{T} D(t, u, r) d \pi_{u} \mid \mathscr{G}_{s}\right], \quad C_{t} \doteq \alpha_{t} \varepsilon_{t}(t, T)
$$

with $0 \leq \alpha_{t} \leq 1$ and where $\alpha_{t}$ is $\mathscr{F}_{t}$-adapted. This means that the close-out is the risk-free mark to market at first default time and the collateral is a fraction $\alpha_{t}$ of the close-out value. An alternative approximation that does not impose a proportionality between the account value processes can be found in [9]. We obtain, by switching to the default-free market filtration $\mathscr{F}$ the following. ${ }^{2}$

Proposition 1 (Master equation under $\mathscr{F}$-conditionally independent default times, no gap risk and $\mathscr{F}_{t}$ measurable payout $\pi_{t}$ ) Under the above assumption, Valuation Equation (2) is further specified as $V_{t}=1_{\{\tau>t\}} \widetilde{V}_{t}$

$$
\begin{aligned}
\widetilde{V}_{t}= & \varepsilon_{t}(t, T)+\mathbb{E}\left[\int_{t}^{T} D(t, u ; r+\lambda)\left(r_{u}-c_{u}\right) \alpha_{u} \varepsilon_{u}(u, T) d u \mid \mathscr{F}_{t}\right] \\
- & \mathbb{E}\left[\int_{t}^{T} D(t, u ; r+\lambda) \lambda_{u}^{C}\left(1-\alpha_{u}\right) L G D_{C}\left(\varepsilon_{u}(u, T)\right)^{+} d u \mid \mathscr{F}_{t}\right] \\
- & \mathbb{E}\left[\int_{t}^{T} D(t, u ; r+\lambda) \lambda_{u}^{I}\left(1-\alpha_{u}\right) L G D_{I}\left(\varepsilon_{u}(u, T)\right)^{-} d u \mid \mathscr{F}_{t}\right]
\end{aligned}
$$

where we introduced the pre-default intensity $\lambda_{t}^{I}$ of the investor and the pre-default intensity $\lambda_{t}^{C}$ of the counterparty as

$$
1_{\left\{\tau_{I}>t\right\}} \lambda_{t}^{I} d t:=\mathbb{Q}\left\{\tau_{I} \in d t \mid \tau_{I}>t, \mathscr{F}_{t}\right\}, 1_{\left\{\tau_{C}>t\right\}} \lambda_{t}^{C} d t:=\mathbb{Q}\left\{\tau_{C} \in d t \mid \tau_{C}>t, \mathscr{F}_{t}\right\}
$$

along with their sum $\lambda_{t}$ and the discount factor for any rate $x_{u}$, namely $D(t, T, x):=$ $\exp \left\{-\int_{t}^{T} x_{u} d u\right\}$.

\section{Valuing Collateralized Interest-Rate Derivatives}

As we mentioned in the introduction, we will base our analysis on real market processes. All liquid market quotes on the money market (MM) correspond to instruments with daily collateralization at overnight rate $\left(e_{t}\right)$, both for the investor and the counterparty, namely $c_{t} \doteq e_{t}$.

\footnotetext{
${ }^{2}$ We refer to [3] and [6] for a precise derivation of the proposition.
} 
Notice that the collateral accrual rate is symmetric, so that we no longer have a dependency of the accrual rates on the collateral price, as opposed to the general master equation case. Moreover, we further assume $r_{t} \doteq e_{t}$.

This makes sense because $e_{t}$ being an overnight rate, it embeds a low counterparty risk and can be considered a good proxy for the risk-free rate $r_{t}$. We will describe some of these MM instruments, such as OIS and Interest Rate Swaps (IRS), along with their underlying market rates, in the following sections. For the remaining of this section we adopt the perfect collateralization approximation of Eq. (1) to derive the valuation equations for OIS and IRS products, hence assuming no gap-risk, while in the numeric experiments of Sect. 4 we will consider also uncollateralized deals. Furthermore, we assume that daily collateralization can be considered as a continuous-dividend perfect collateralization. See [4] for a discussion on the impact of discrete-time collateralization on interest-rate derivatives.

\subsection{Overnight Rates and OIS}

Among other instruments, the MM usually quotes the prices of overnight indexed swaps (OIS). Such contracts exchange a fix-payment leg with a floating leg paying a discretely compounded rate based on the same overnight rate used for their collateralization. Since we are going to price OIS under the assumption of perfect collateralization, namely we are assuming that daily collateralization may be viewed as done on a continuous basis, we approximate also daily compounding in OIS floating leg with continuous compounding, which is reasonable when there is no gap risk. Hence the discounted payoff of a one-period OIS with tenor $x$ and maturity $T$ is given by

$$
D(t, T, e)\left(1+x K-\exp \left\{\int_{T-x}^{T} e_{u} d u\right\}\right)
$$

where $K$ is the fixed rate payed by the OIS. Furthermore, we can introduce the (par) fix rates $K=E_{t}(T, x ; e)$ that make the one-period OIS contract fair, namely priced 0 at time $t$. They are implicitly defined via

$$
\widetilde{V}_{t}^{\mathrm{OIS}}(K):=\mathbb{E}\left[\left(1+x K-\exp \left\{\int_{T-x}^{T} e_{u} d u\right\}\right) D(t, T ; e) \mid \mathscr{F}_{t}\right]
$$

with $\widetilde{V}_{t}^{\mathrm{OIS}}\left(E_{t}(T, x ; e)\right)=0$ leading to

$$
E_{t}(T, x ; e):=\frac{1}{x}\left(\frac{P_{t}(T-x ; e)}{P_{t}(T ; e)}-1\right)
$$


where we define collateralized zero-coupon bonds ${ }^{3}$ as

$$
P_{t}(T ; e):=\mathbb{E}\left[D(t, T ; e) \mid \mathscr{F}_{t}\right]
$$

One-period OIS rates $E_{t}(T, x ; e)$, along with multi-period ones, are actively traded on the market. Notice that we can bootstrap collateralized zero-coupon bond prices from OIS quotes.

\subsection{LIBOR Rates, IRS and Basis Swaps}

LIBOR rates $\left(L_{t}(T)\right)$ used to be linked to the term structure of default-free interlink interest rates in a fundamental way. In the classical term structure theory, LIBOR rates would satisfy fundamental no-arbitrage conditions with respect to zero-coupon bonds that we no longer consider to hold, as we pointed out earlier in (1). We now deal with a new definition of forward LIBOR rates that may take into account collateralization. LIBOR rates are still the indices used as reference rate for many collateralized interest-rate derivatives (IRS, basis swaps, ...). IRS contracts swap a fix-payment leg with a floating leg paying simply compounded LIBOR rates. IRS contracts are collateralized at overnight rate $e_{t}$. Thus, a discounted one-period IRS payoff with maturity $T$ and tenor $x$ is given by

$$
D(t, T, e) x\left(K-L_{T-x}(T)\right)
$$

where $K$ is the fix rate payed by the IRS. Furthermore, we can introduce the (par) fix rates $K=F_{t}(T, x ; e)$ that render the one-period IRS contract fair, i.e. priced at zero. They are implicitly defined via

$$
\widetilde{V}_{t}^{\mathrm{IRS}}(K):=\mathbb{E}\left[\left(x K-x L_{T-x}(T)\right) D(t, T ; e) \mid \mathscr{F}_{t}\right]
$$

with $\widetilde{V}_{t}^{\mathrm{IRS}}\left(F_{t}(T, x ; e)\right)=0$, leading to the following definition of forward LIBOR rate

$$
F_{t}(T, x ; e):=\frac{\mathbb{E}\left[L_{T-x}(T) D(t, T ; e) \mid \mathscr{F}_{t}\right]}{\mathbb{E}\left[D(t, T ; e) \mid \mathscr{F}_{t}\right]}=\frac{\mathbb{E}\left[L_{T-x}(T) D(t, T ; e) \mid \mathscr{F}_{t}\right]}{P_{t}(T ; e)}
$$

The above definition may be simplified by a suitable choice of the measure under which we take the expectation. In particular, we can consider the following Radon-Nikodym derivative, defining the collateralized $T$-forward measure $\mathbb{Q}^{T ; e}$,

\footnotetext{
${ }^{3}$ Notice that we are only defining a price process for hypothetical collateralized zero-coupon bond. We are not assuming that collateralized bonds are assets traded on the market.
} 


$$
Z_{t}(T ; e):=\left.\frac{d \mathbb{Q}^{T ; e}}{d \mathbb{Q}}\right|_{\mathscr{F}_{t}}:=\frac{\mathbb{E}\left[D(0, T ; e) \mid \mathscr{F}_{t}\right]}{P_{0}(T ; e)}=\frac{D(0, t ; e) P_{t}(T ; e)}{P_{0}(T ; e)}
$$

which is a positive $\mathbb{Q}$-martingale, normalized so that $Z_{0}(T ; e)=1$.

Thus, for any payoff $\phi_{T}$, perfectly collateralized at overnight rate $e_{t}$, we can express prices as expectations under the collateralized $T$-forward measure and in particular, we can write LIBOR forward rates as

$$
F_{t}(T, x ; e):=\frac{\mathbb{E}\left[L_{T-x}(T) D(t, T ; e) \mid \mathscr{F}_{t}\right]}{\mathbb{E}\left[D(t, T ; e) \mid \mathscr{F}_{t}\right]}=\mathbb{E}^{T ; e}\left[L_{T-x}(T) \mid \mathscr{F}_{t}\right]
$$

One-period forward rates $F_{t}(T, x ; e)$, along with multi-period ones (swap rates), are actively traded on the market. Once collateralized zero-coupon bonds are derived, we can bootstrap forward rate curves from such quotes. See, for instance, [1] or [27] for a discussion on bootstrapping algorithms.

Basis swaps are an interesting product that became more popular after the market switched to a multi-curve structure. In fact, in a basis swap there are two floating legs, one pays a LIBOR rate with a certain tenor and the other pays the LIBOR rate with a shorter tenor plus a spread that makes the contract fair at inception. More precisely, the payoff of a basis swap whose legs pay respectively a LIBOR rate with tenors $x<y$ with maturity $T=n x=m y$ is given by

$$
\begin{aligned}
& \sum_{i=1}^{n} D(t, T-(n-i) x, e) x\left(L_{T-(n-i-1) x}(T-(n-i) x)+K\right) \\
& \quad-\sum_{j=1}^{m} D(t, T-(m-j) y, e) y L_{T-(m-j-1) y}(T-(m-j) y) .
\end{aligned}
$$

It is clear that apart from being traded per se, this instrument is naturally present in the banks portfolios as result of the netting of opposite swap positions with different tenors.

\subsection{Modeling Constraints}

Our aim is to set up a multiple-curve dynamical model starting from collateralized zero-coupon bonds $P_{t}(T ; e)$, and LIBOR forward rates $F_{t}(T, x ; e)$. As we have seen we can bootstrap the initial curves for such quantities from directly observed quotes in the market. Now, we wish to propose a dynamics that preserves the martingale properties satisfied by such quantities. Thus, without loss of generality, we can define collateralized zero-coupon bonds under the $\mathbb{Q}$ measure as

$$
d P_{t}(T ; e)=P_{t}(T ; e)\left(e_{t} d t-\sigma_{t}^{P}(T ; e)^{*} d W_{t}^{e}\right)
$$


and LIBOR forward rates under the $\mathbb{Q}^{T ; e}$ measure as

$$
d F_{t}(T, x ; e)=\sigma_{t}^{F}(T, x ; e)^{*} d Z_{t}^{T ; e}
$$

where $W^{e}$ and $Z^{T ; e}$ are correlated standard (column) vector ${ }^{4}$ Brownian motions with correlation matrix $\rho$, and the volatility vector processes $\sigma^{P}$ and $\sigma^{F}$ may depend on bonds and forward LIBOR rates themselves.

The following definition of $f_{t}(T, e)$ is not strictly necessary, and we could keep working with bonds $P_{t}(T ; e)$, using their dynamics. However, as it is customary in interest rate theory to model rates rather than bonds, we may try to formulate quantities that are closer to the standard HJM framework. In this sense we can define instantaneous forward rates $f_{t}(T ; e)$, by starting from (collateralized) zero-coupon bonds, as given by

$$
f_{t}(T ; e):=-\partial_{T} \log P_{t}(T ; e)
$$

We can derive instantaneous forward-rate dynamics by Itô lemma, and we obtain the following dynamics under the $\mathbb{Q}^{T ; e}$ measure

$$
d f_{t}(T ; e)=\sigma_{t}(T ; e) d W_{t}^{T ; e}, \quad \sigma_{t}(T ; e):=\partial_{T} \sigma_{t}^{P}(T ; e)
$$

where the $W^{T ; e} \mathrm{~S}$ are Brownian motions and partial differentiation is meant to be applied component-wise.

Hence, we can summarize our modeling assumptions in the following way. Since linear products (OIS, IRS, basis swaps...) can be expressed in terms of simpler quantities, namely collateralized zero-coupon bonds $P_{t}(T ; e)$ and LIBOR forward rates $F_{t}(T, x ; e)$, we focus on their modeling. The initial term structures for collateralized products may be bootstrapped from market data, and for volatility and dynamics, we can write rates dynamics by enforcing suitable no-arbitrage martingale properties, namely

$$
d f_{t}(T ; e)=\sigma_{t}(T ; e) \cdot d W_{t}^{T ; e}, d F_{t}(T, x ; e)=\sigma_{t}^{F}(T, x ; e) \cdot d Z_{t}^{T ; e} .
$$

As we explained in the introduction, this is where the multiple curve picture finally shows up: we have a curve with LIBOR-based forward rates $F_{t}(T, x ; e)$, that are collateral adjusted expectation of LIBOR market rates $L_{T-x}(T)$ we take as primitive rates from the market, and we have instantaneous forward rates $f_{t}(T ; e)$ that are OIS-based rates. OIS rates $f_{t}(T ; e)$ are driven by collateral fees, whereas LIBOR forward rates $F_{t}(T, x ; e)$ are driven both by collateral rates and by the primitive LIBOR market rates.

\footnotetext{
${ }^{4}$ In the following we will consider $N$-dimensional vectors as $N \times 1$ matrices. Moreover, given a matrix $A$, we will indicate $A^{*}$ its transpose, and if $B$ is another conformable matrix we indicate $A B$ the usual matrix product.
} 


\section{Interest-Rate Modeling}

We can now specialize our modeling assumptions to define a model for interest-rate derivatives which is on one hand flexible enough to calibrate the quotes of the MM, and on the other hand robust. Our aim is to use an HJM framework using a single family of Markov processes to describe all the term structures and interest rate curves we are interested in.

In the literature many authors proposed generalizations of the HJM framework to include multiple yield curves. In particular, we cite the works of [12-14, 16, 20-23]. A survey of the literature can be found in [17].

In such works the problem is faced in a pragmatic way by considering each forward rate as a single asset without investigating the microscopical dynamics implied by liquidity and credit risks. However, the hypothesis of introducing different underlying assets may lead to over-parametrization issues that affect the calibration procedure. Indeed, the presence of swap and basis-swap quotes on many different yield curves is not sufficient, as the market quotes swaption premia only on few yield curves. For instance, even if the Euro market quotes one-, three-, six- and twelve-month swap contracts, liquidly traded swaptions are only those indexed to the three-month (maturity one-year) and the six-month (maturities from two to thirty years) Euribor rates. Swaptions referring to other Euribor tenors or to overnight rates are not actively quoted.

In order to solve such problem [23] introduces a parsimonious model to describe a multi-curve setting by starting from a limited number of (Markov) processes, so as to extend the logic of the HJM framework to describe with a unique family of Markov processes all the curves we are interested in.

\subsection{Multiple-Curve Collateralized HJM Framework}

We follow $[22,23]$ by reformulating their theory under the $\mathbb{Q}^{T ; e}$ measure. We model only observed rates as in market model approaches and we consider a common family of processes for all the yield curves of a given currency, so that we are able to build parsimonious yet flexible models. Hence let us summarize the basic requirements the model must fulfill:

(i) existence of OIS rates, which we can describe in terms of instantaneous forward rates $f_{t}(T ; e)$

(ii) existence of LIBOR rates assigned by the market, typical underlyings of traded derivatives, with associated forwards $F_{t}(T, x ; e)$;

(iii) no arbitrage dynamics of the $f_{t}(T ; e)$ and the $F_{t}(T, x ; e)$ (both being $(T, e)$ forward measure martingales);

(iv) possibility of writing both $f_{t}(T ; e)$ and $F_{t}(T, x ; e)$ as functions of a common family of Markov processes, so that we are able to build parsimonious yet flexible models. 
While the first two points are related to the set of financial quantities we are about to model, the last two are conditions we impose on their dynamics, and will be granted by the right choice of model volatilities. Hence, we choose under $\mathbb{Q}^{T ; e}$ measure, the following dynamics:

$$
\begin{aligned}
d f_{t}(T ; e) & =\sigma_{t}(T)^{*} d W_{t}^{T ; e} \\
d F_{t}(T, x ; e) & =\left(k(T, x)+F_{t}(T, x ; e)\right) \Sigma_{t}(T, x)^{*} d W_{t}^{T ; e}
\end{aligned}
$$

where we introduce the families of (stochastic $N$-dimensional) volatility processes $\sigma_{t}(T)$ and $\Sigma_{t}(T, x)$, the vector of $N$ independent $\mathbb{Q}^{T ; e}$-Brownian motions $W_{t}^{T ; e}$, and the set of deterministic shifts $k(T, x)$, such that $\lim _{x \rightarrow 0} x k(T, x)=1$. This limit condition ensures that the model approaches a standard default- and liquidity-free HJM model when the tenor goes to zero. We bootstrap $f_{0}(T ; e)$ and $F_{0}(T, x ; e)$ from market quotes.

In order to get a model with a reduced number of common driving factors in the spirit of HJM approaches, it is sufficient to conveniently tie together the volatility processes $\sigma_{t}(T)$ and $\Sigma_{t}(T, x)$ through a third volatility process $\sigma_{t}(u, T, x)$.

$$
\sigma_{t}(T):=\sigma_{t}(T ; T, 0), \quad \Sigma_{t}(T, x):=\int_{T-x}^{T} \sigma_{t}(u ; T, x) d u .
$$

Under this parametrization the OIS curve dynamics is the very same as the riskfree curve in an ordinary HJM framework. Indeed, we have for linearly compounding forward rates

$$
d E_{t}(T, x ; e)=\left(1 / x+E_{t}(T, x ; e)\right) \int_{T-x}^{T} \sigma_{t}(u)^{*} d u d W_{t}^{T ; e} .
$$

In the generalized version of the HJM framework proposed by [23] we have an explicit expression for both the collateralized zero-coupon bonds $P_{t}(T ; e)$ and the LIBOR forward rates $F_{t}(T, x ; e)$. The first result is a direct consequence of modeling the OIS curve as the risk-free curve in a standard HJM framework, while the second result can be achieved only if a particular form of the volatilities is selected. We obtain this if we generalize the approach of [28] by introducing the following separability constraint

$$
\begin{aligned}
\sigma_{t}(u, T, x) & :=h(t) q(u, T, x) g(t, u), \\
g(t, u) & :=\exp \left\{-\int_{t}^{u} a(s) d s\right\}, \quad q(u ; u, 0):=I d,
\end{aligned}
$$

where $h_{t}$ is an $N \times N$ matrix process, $q(u, T, x)$ is a deterministic $N \times N$ diagonal matrix function, and $a(s)$ is a deterministic $N$-dimensional vector function. The condition on $q(u ; T, x)$ being the identity matrix, when $T=u$ ensures that a standard HJM framework holds for collateralized zero-coupon bonds. 
We can work out an explicit expression for the LIBOR forward rates, by plugging the expression of the volatilities into Eq. (7). We obtain

$$
\begin{aligned}
& \log \left(\frac{k(T, x)+F_{t}(T, x ; e)}{k(T, x)+F_{0}(T, x ; e)}\right) \\
& \quad=G(t, T-x, T ; T, x)^{*}\left(X_{t}+Y_{t}\left(G_{0}(t, t, T)-\frac{1}{2} G(t, T-x, T ; T, x)\right)\right),
\end{aligned}
$$

where the stochastic vector process $X_{t}$ and the auxiliary matrix process $Y_{t}$ are defined under the $\mathbb{Q}$ measure as in the ordinary HJM framework

$$
\begin{aligned}
X_{t}^{i} & =\sum_{k=1}^{N} \int_{0}^{t} g_{i}(s, t)\left(h_{i k, s} d W_{k, s}+\left(h_{s}^{*} h_{s}\right)_{i k} \int_{s}^{t} d y g_{k}(s, y) d s\right) \quad, i=1 \ldots N \\
Y_{t}^{i k} & =\int_{0}^{t} g_{i}(s, t)\left(h_{s}^{*} h_{s}\right)_{i k} g_{k}(s, t) d s \quad i, k=1 \ldots N
\end{aligned}
$$

and

$$
G_{0}\left(t, T_{0}, T_{1}\right)=\int_{T_{0}}^{T_{1}} g(t, s) d s, \quad G\left(t, T_{0}, T_{1}, T, x\right)=\int_{T_{0}}^{T_{1}} q(s, T, x) g(t, s) d s .
$$

It is worth noting that the integral representation of forward LIBOR volatilities given by Eq. (8), together with the common separability constraint given in Eq. (9) are sufficient conditions to ensure the existence of a reconstruction formula for all OIS and LIBOR forward rates based on the very same family of Markov processes (see [3]).

We are interested in some specification of this model, in particular a variant of the Hull and White model (HW), a variant of the Cheyette model (Ch) and the Moreni and Pallavicini model (MP). The HW model [18] is the simplest one, and is obtained choosing

$$
h(t) \doteq R, \quad q(u, T, x) \doteq I d, \quad a(s) \doteq a, \quad \kappa(T, x) \doteq \frac{1}{x}
$$

where $a$ is a constant vector, and $R$ is the Cholesky decomposition of the correlation matrix that we want our $X_{t}$ vector to have. In this case we obtain $\sigma_{t}(u ; T, x)=$ $R \cdot e^{-a(u-t)}$, where the exponential is intended to be component-wise. Then we note that $X_{t}$ is a mean reverting Gaussian process while the $Y_{t}$ process is deterministic.

In order to model implied volatility smiles, we can add a stochastic volatility process to our model, as shown in [22]. In particular we can obtain a variant of the Ch model ([10]), considering a common square-root process for all the entries of $h$, as in [29]. More precisely we replace $h(t)$ in (11) with $h(t) \doteq \sqrt{v_{t}} R$. With $a$ and $R$ as before and $v_{t}$ being a process with the following dynamic: 


$$
d v_{t}=\eta\left(1-v_{t}\right) d t+v_{0}\left(1+\left(v_{1}-1\right) e^{-v_{2} t}\right) \sqrt{v_{t}} d Z_{t}, \quad v_{0}=\bar{v}
$$

where $Z_{t}$ is a Brownian motion correlated to $W_{t}$. Obtaining as a volatility process $\sigma_{t}(u ; T, x)=\sqrt{v_{t}} R \cdot e^{-a(u-t)}$.

As the last specification of the framework we consider the MP model which uses a different shift $k(T, x)$, and introduces a dependence on the tenor in the volatility process.

$$
h(t) \doteq \sqrt{v_{t}} R, \quad q(u, T, x)^{i, i} \doteq e^{x \eta^{i}}, \quad a(s) \doteq a, \quad \kappa(T, x) \doteq \frac{e^{-\gamma x}}{x}
$$

With $a$ and $R$ as before and $v_{t}$ being defined by (12). Here we have for the volatility $\sigma_{t}(u ; T, x)=\sqrt{v_{t}} R \cdot e^{\eta x-a(u-t)}$.

To better appreciate the difference between the $\mathrm{Ch}$ model and the MP model one could compute the quantity

$$
\beta_{t}\left(x_{1}, x_{2} ; e\right):=\frac{1}{x_{2}} \log \left(\frac{\frac{1}{x_{2}}+E_{t}\left(t+x_{2}, x_{2} ; e\right)}{\frac{1}{x_{2}}+F_{t}\left(t+x_{2}, x_{2} ; e\right)}\right)-\frac{1}{x_{1}} \log \left(\frac{\frac{1}{x_{1}}+E_{t}\left(t+x_{1}, x_{1} ; e\right)}{\frac{1}{x_{1}}+F_{t}\left(t+x_{1}, x_{1} ; e\right)}\right)
$$

which represents the time-normalized difference between two forward rates with different tenors and thus can be used as a proxy for the value of a basis swap. We have that in the HW and in the Ch models $\beta_{t}\left(x_{1}, x_{2} ; e\right)$ is deterministic while in the MP model is a stochastic quantity. This suggests that the MP model should be able to better capture the dynamics of the basis between two rates with different tenors. We refer the reader to [3] for a more detailed analysis of the issue, and to [23] for calibration and valuation examples for the swaptions and cap/floor market.

\subsection{Numerical Results}

We apply our framework to simple but relevant products: an IRS and a basis swap. We analyze the impact of the choice of an interest rate model on the portfolio valuation, in particular we measure the dependency of the price on the correlations between interest-rates and credit spreads, the so-called wrong-way risk. We model the market risks by simulating the following processes in a multiple-curve HJM model under the pricing measure $\mathbb{Q}$. The overnight rate $e_{t}$ and the LIBOR forward rates $F_{t}(T ; e)$ are simulated according to the dynamics given in Sect.4.1. Maintaining the same notation of the aforementioned section, we choose $N=2$, and for our numerical experiments we use a HW model, a Ch model and an MP model, all calibrated to swaption at-the-money volatilities listed on the European market.

As we have already noted, the Ch model introduces a stochastic volatility and hence has an increased number of parameters with respect to the HW model. The MP model aims at better modeling the basis between rates with different tenors, while keeping the model parsimonious in terms of extra parameters with respect to the $\mathrm{Ch}$ 
model. In particular the HW model is able to reproduce the ATM quotes but is not able to correctly reproduce the volatility smile. On the other hand, the introduction of a stochastic volatility process helps in recovering the market data smile and thus the $\mathrm{Ch}$ and the MP models have similar results in properly fitting the smile. The detailed results of the calibration are available in [3].

For what concerns the credit part, the default intensities of the investor and the counterparty are given by two CIR++ processes $\lambda_{t}^{i}=y_{t}^{i}+\psi^{i}(t)$ under the $\mathbb{Q}^{T ; e}$ measure, i.e. they follow

$$
d y_{t}^{i}=\gamma^{i}\left(\mu^{i}-y_{t}^{i}\right) d t+\zeta^{i} \sqrt{y_{t}^{i}} d Z_{t}^{i}, \quad i \in\{I, C\}
$$

where the two $Z^{i}$ s are Brownian motions correlated with the $W^{T ; e} \mathrm{~s}$, and they are calibrated to the market data shown in [4]. In particular, two different market settings are used in the numerical examples: the medium risk and the high risk settings. The correlations among the risky factors are induced by correlating the Brownian motions as in [8].

We now analyze the impact of wrong-way risk on the bilateral adjustment, namely CVA plus DVA, of IRS and basis swaps when collateralization is switched off, namely we want to evaluate Eq. (1) when $\alpha_{t} \doteq 0$. For an extended analysis see [3]. Wrongway risk is expressed with respect to the correlation between the default intensities and a proxy of market risk, namely the short rate $e_{t}$.

In Fig. 1 we show the variation of the bilateral adjustment for a ten years IRS receiving a fix rate yearly and paying $6 \mathrm{~m}$ Libor twice a year and for a ten years basis swap receiving $3 \mathrm{~m}$ Libor plus spread and paying $6 \mathrm{~m}$ Libor. It is clear that for a product like the IRS, not subject to the basis dynamic, we have that the big difference among the models is the presence of a stochastic volatility. In fact we can see that the $\mathrm{Ch}$ model and the MP model are almost indistinguishable while the results of the HW model are different from the stochastic volatility ones. Moreover we can
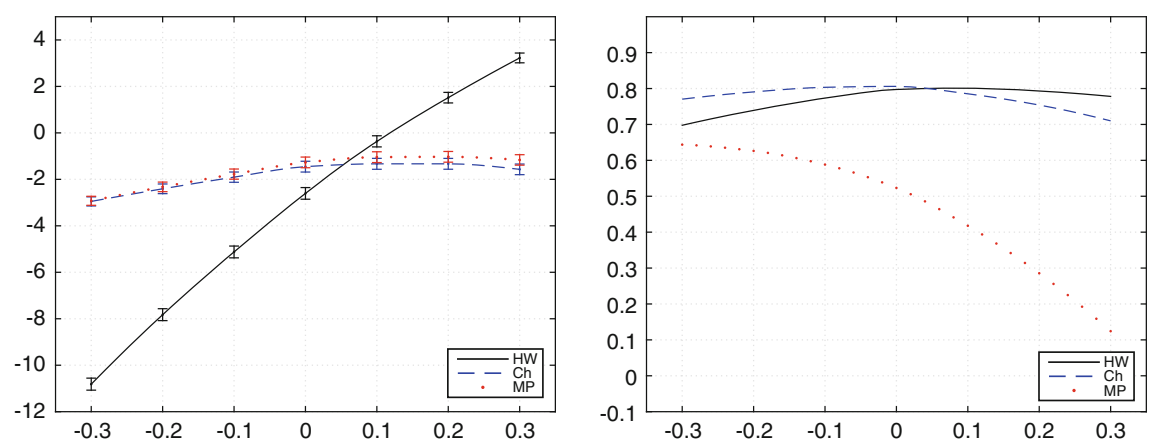

Fig. 1 Wrong-way risk for different models. On the horizontal axis correlation among credit and market risks; on the vertical axis the bilateral adjustment, namely CVA + DVA, in basis points. Left panel a 10y IRS receiving a fix rate and paying $6 \mathrm{~m}$ Libor. Right panel a 10y basis swap receiving $3 \mathrm{~m}$ Libor plus spread and paying $6 \mathrm{~m}$ Libor. Montecarlo error is displayed where significant 
observe that all the models have the same trend, i.e. the bilateral adjustment grows as correlation increase. In fact this can be explained by the fact that a higher correlation means that the deal will be more profitable when it will be more risky (since we are receiving the fixed rate and paying the floating one), hence the bilateral adjustment will be bigger.

In the case of a basis swap instead, we see that, as said before, the HW model and the $\mathrm{Ch}$ model do not have a basis dynamic and hence the curve represented is almost flat. On the other hand the MP model is able to capture the dynamics of the basis and hence we can see that the more the overnight rate is correlated with the credit risk the smaller the bilateral adjustment becomes.

We conclude by pointing out that our analysis will be extended to partially collateralized deals in future work. In such a context funding costs enter the picture in a more comprehensive way. Some initial suggestions in this respect were given in [24].

Acknowledgements The KPMG Center of Excellence in Risk Management is acknowledged for organizing the conference "Challenges in Derivatives Markets - Fixed Income Modeling, Valuation Adjustments, Risk Management, and Regulation".

Open Access This chapter is distributed under the terms of the Creative Commons Attribution 4.0 International License (http://creativecommons.org/licenses/by/4.0/), which permits use, duplication, adaptation, distribution and reproduction in any medium or format, as long as you give appropriate credit to the original author(s) and the source, a link is provided to the Creative Commons license and any changes made are indicated.

The images or other third party material in this chapter are included in the work's Creative Commons license, unless indicated otherwise in the credit line; if such material is not included in the work's Creative Commons license and the respective action is not permitted by statutory regulation, users will need to obtain permission from the license holder to duplicate, adapt or reproduce the material.

\section{References}

1. Ametrano, F.M., Bianchetti, M.: Bootstrapping the illiquidity: multiple yield curves construction for market coherent forward rates estimation. In: Mercurio, F. (ed.) Modeling Interest Rates: Latest Advances for Derivatives Pricing. Risk Books, London (2009)

2. Bielecki, T., Rutkowski, M.: Credit Risk: Modeling, Valuation and Hedging. Springer Finance. Springer, Berlin (2002)

3. Bormetti, G., Brigo, D., Francischello, M., Pallavicini, A.: Impact of multiple curve dynamics in credit valuation adjustments under collateralization. arXiv preprint arXiv:1507.08779 (2015)

4. Brigo, D., Capponi, A., Pallavicini, A., Papatheodorou, V.: Collateral margining in arbitragefree counterparty valuation adjustment including re-hypothecation and netting. arXiv preprint arXiv:1101.3926 (2011)

5. Brigo, D., Capponi, A., Pallavicini, A.: Arbitrage-free bilateral counterparty risk valuation under collateralization and application to credit default swaps. Math. Financ. 24(1), 125-146 (2014)

6. Brigo, D., Francischello, M., Pallavicini, A.: Invariance, existence and uniqueness of solutions of nonlinear valuation PDEs and FBSDEs inclusive of credit risk, collateral and funding costs. arXiv preprint arXiv:1506.00686 (2015). A refined version of this report by the same authors is being published in this same volume 
7. Brigo, D., Liu, Q., Pallavicini, A., Sloth, D.: Nonlinear valuation under collateral, credit risk and funding costs: a numerical case study extending Black-Scholes. arXiv preprint arXiv:1404.7314 (2014). A refined version of this report by the same authors is being published in this same volume

8. Brigo, D., Pallavicini, A.: Counterparty risk under correlation between default and interest rates. In: Miller, J., Edelman, D., Appleby, J. (eds.) Numerical Methods for Finance. Chapman Hall, Atlanta (2007)

9. Brigo, D., Pallavicini, A.: Nonlinear consistent valuation of CCP cleared or CSA bilateral trades with initial margins under credit, funding and wrong-way risks. J. Financ. Eng. 1(1), 1-61 (2014)

10. Cheyette, O.: Markov representation of the Heath-Jarrow-Morton model. Available at SSRN $6073(1992)$

11. Crépey, S., Gerboud, R., Grbac, Z., Ngor, N.: Counterparty risk and funding: the four wings of theTVA. Int. J. Theor. Appl. Financ. 16(2), 1350006-1-1350006-31 (2013)

12. Crépey, S., Grbac, Z., Nguyen, H.: A multiple-curve HJM model of interbank risk. Math. Financ. Econ. 6(3), 155-190 (2012)

13. Crépey, S., Grbac, Z., Ngor, N., Skovmand, D.: A Lévy HJM multiple-curve model with application to CVA computation. Quant. Financ. 15(3), 401-419 (2015)

14. Cuchiero, C., Fontana, C., Gnoatto, A.: A general HJM framework for multiple yield curve modeling. Financ. Stoch. 20(2), 267-320 (2016)

15. Filipović, D., Trolle, A.B.: The term structure of interbank risk. J. Financ. Eng. 109(3), 707-733 (2013)

16. Fujii, M., Takahashi, A.: Derivative pricing under asymmetric and imperfect collateralization and CVA. Quant. Financ. 13(5), 749-768 (2013)

17. Henrard, M.: Interest Rate Modelling in the Multi-Curve Framework: Foundations, Evolution and Implementation. Palgrave Macmillan, Basingstoke (2014)

18. Hull, J., White, A.: Pricing interest-rate-derivative securities. Rev. Financ. Stud. 3(4), 573-592 (1990)

19. Mercurio, F.: Interest rates and the credit crunch: New formulas and market models. Bloomberg Portfolio Research Paper (2009)

20. Mercurio, F.: LIBOR market models with stochastic basis. Risk Mag. 23(12), 84-89 (2010)

21. Mercurio, F., Xie, Z.: The basis goes stochastic. Risk Mag. 23(12), 78-83 (2012)

22. Moreni, N., Pallavicini, A.: Parsimonious multi-curve HJM modelling with stochastic volatility. In: Bianchetti, M., Morini, M. (eds.) Interest Rate Modelling After the Financial Crisis. Risk Books, London (2013)

23. Moreni, N., Pallavicini, A.: Parsimonious HJM modelling for multiple yield curve dynamics. Quant. Financ. 14(2), 199-210 (2014)

24. Pallavicini, A., Brigo, D.: Interest-rate modelling in collateralized markets: multiple curves, credit-liquidity effects, CCPs. arXiv preprint arXiv:1304.1397 (2013)

25. Pallavicini, A., Perini, D., Brigo, D.: Funding valuation adjustment: FVA consistent with CVA, DVA, WWR, collateral, netting and re-hyphotecation. arXiv preprint arXiv:1112.1521 (2011)

26. Pallavicini, A., Perini, D., Brigo, D.: Funding, collateral and hedging: uncovering the mechanics and the subtleties of funding valuation adjustments. arXiv preprint arXiv:1210.3811 (2012)

27. Pallavicini, A., Tarenghi, M.: Interest-rate modelling with multiple yield curves. Available at SSRN 1629688 (2010)

28. Ritchken, P., Sankarasubramanian, L.: Volatility structures of forward rates and the dynamics of the term structure. Math. Financ. 7, 157-176 (1995)

29. Trolle, A., Schwartz, E.: A general stochastic volatility model for the pricing of interest rate derivatives. Rev. Financ. Stud. 22(5), 2007-2057 (2009) 